\title{
Numerical simulation of expansion of the cavity in tube-wall based on tube transportation of ultra-critical $\mathrm{CO}_{2}$
}

\author{
Weihao Wang ${ }^{1, a}$, Chen Wang 2,b \\ ${ }^{1}$ Class 10 grade three, The high school affiliated to Harbin Normal University, Heilongjiang, Harbin, \\ 150080, China \\ ${ }^{2}$ School of Civil Engineering, Harbin Institute of Technology, Heilongjiang, Harbin, 150001, China \\ ${ }^{a}$ E-mail address:hitwangchen@163.com
}

Keywords: GRP pipe; ultra-critical $\mathrm{CO}_{2}$; the cavity; expansion

Abstract. The paper simulated the mechanical process of transportation of ultra-critical $\mathrm{CO}_{2}$ using glass reinforced plastic pipe (GRP pipe). The gas expansion model of micro-cavity in GRP pipe was established based on the experimental results. By analyzing the physical process of gas expansion on the micro-pore, the computer simulation method was used to reveal the law of the stress and strain distribution in the tuber-wall. The results provided the basic research on the technology application of the transportation of supercritical pipe.

\section{Introduction}

In the petrochemical industry field, the $\mathrm{CO}_{2}$ flood technology is a kind of more advanced technology. The technology started in American in1973 at the Mississippi, following by Wenbury oil of Canada, North Sea oil of Britain and so on. The long distance and large diameter pipe transportation of ultra-critical $\mathrm{CO}_{2}$ is the inevitable developing trend in the future. The technology solved also the worldwide problem on greenhouse emission. However, the technology of $\mathrm{CO}_{2}$ phase or ultra-critical injection is still an issue to solve for applying on the long distant transport industry. Currently, the injecting $\mathrm{CO}_{2}$ technology is usually used for difficultly mining crude oil, and the injection pressure is about $10 \sim 18 \mathrm{MPa}$, that is, the super-critical level of well head pressure. Considering the $\mathrm{CO}_{2}$ soluble in water and oil, the $\mathrm{CO}_{2}$ injection can improve its solubility, and then increase the bulk and decreased the density of crude oil correspondingly. The technology can improve effectively the extract rate of oil by increasing the activity of oil layers and the flowability of crude oil.

Studies show that filament winding layer of GRP pipe usually exists the cavity and other flaws[1,3]. Therefore, the High-pressure $\mathrm{CO}_{2}$ would penetrate laminated structure and accumulate in the cavity when the tube loads the continuous pressure. When the pressure of piping transport lasted for a proper time, the internal pressure on the cavity will tend to the balance[4,7]. Based on the above facts, the paper established the model, simulated the work process of the transport pipe and the developing law of expansion of the cavity in the laminated pipe. It can provide theoretical basis on high-pressure $\mathrm{CO}_{2}$ injection pipeline system application.

\section{Mechanical modeling}

Fig.1 shows the cavity of GRP (curing be the aromatic amine) pipe under the SEM. When the transport pressure reached the ultra-critical $\mathrm{CO}_{2}$, the $\mathrm{CO}_{2}$ would penetrate laminated structure, accumulate in the cavity and balanced at the constant value. For meeting the production requirements, the conveying substance and pressure were always changing in the transporting pipe. Therefore, these would lead to change of the tube-wall pressure, correspondingly. However, the $\mathrm{CO}_{2}$ of penetrating and accumulating in the cavity couldn't release immediately and remain the balance pressure, but the pressure in the pipe decreased promptly. Therefore, the balance between the piping pressure and the tube-wall cavity pressure will be destroyed. So, the $\mathrm{CO}_{2}$ of the micro-pore will expand and lead to the elastic-plastic deformation of laminated materials and increase the volume of the cavity (see fig.2). 


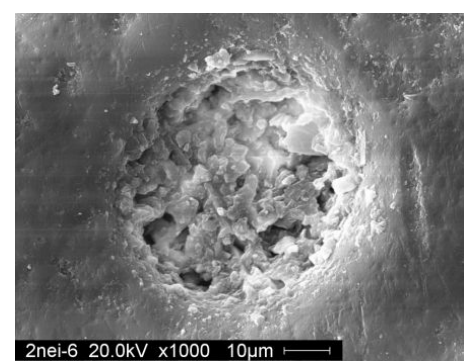

Fig.1 The cavity of layup

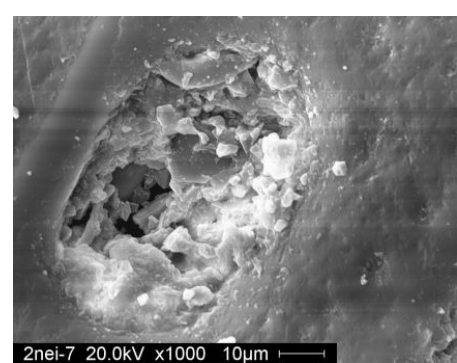

Fig.2 Cavity deformation by $\mathrm{CO}_{2}$ expansion

When the cavity expansion exceeded the strength or deformation of matrix materials, the more plastic deformation will cause the matrix materials failure near the cavity. By the injection $\mathrm{CO}_{2}$ high-pressure test, the results show that the laminated cavity will occurred the larger deformation caused by the expansion. Fig. 3 is the laminated cavity after keeping the pressure for a while.

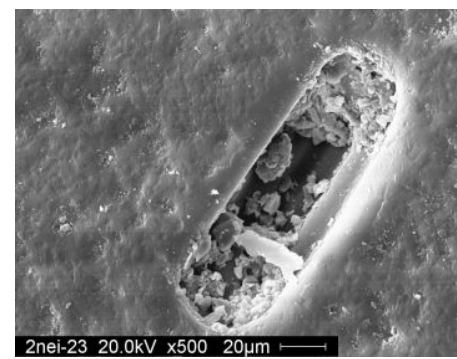

Fig.3 Laminated cavity

On the basis of manufacturing processes, material composition and the structure character of pipe, the pipe was made by winding fiber laminates in $\pm 30^{\circ}$ direction. Fig .5 is the mechanical model of the pipe.

The geometry size of pipe: the thickness both laminates and matrix is $1 \mathrm{~mm}$, the in-diameter and ex-diameter is $38 \mathrm{~mm}, 48 \mathrm{~mm}$ respectively.

The materials of the pipe: the elastic modulus is $22.8 \mathrm{GPa}$, the axial elastic modulus is $12.6 \mathrm{GPa}$, Poisson's ratio is 0.38 , the max transporting pressure is $17 \mathrm{MPa}$.

Assuming the tube-wall existing a cavity and the cavity was simplified the sphere and its diameter is $\mathrm{D}=1 \mathrm{~mm}$. fig. 6 is the model of pipe with cavity in the tube-wall.

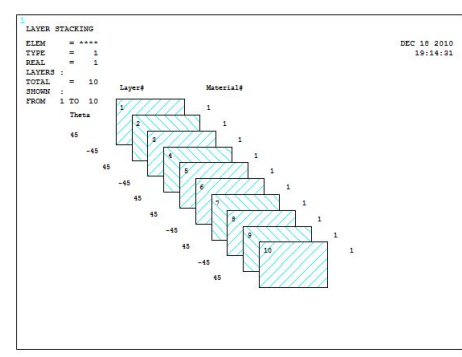

Fig.4 Laminated structure of tube wall

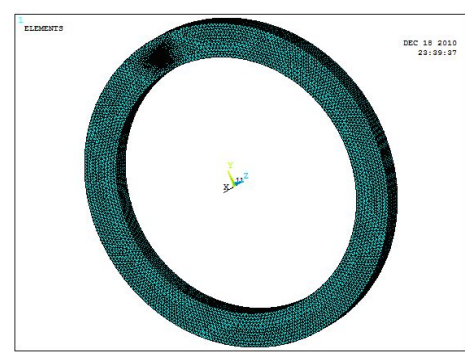

Fig.5 The model of pipe with cavity

The model detail and the meshing of the model is shown in Fig.7

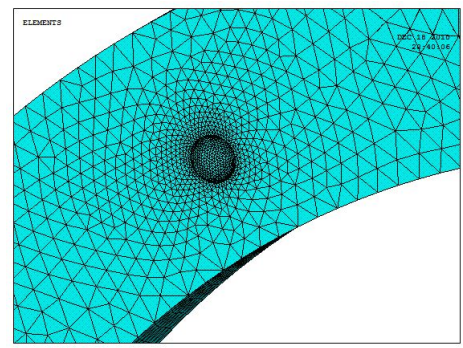

Fig.6 Cavity and the meshing

For the spherical cavity forming by aeration porosity in the tube wall, when the pressure of the tube decreased sharply, the balance of the in- and ex- tube wall would be destroyed and lead to 
expand of the gas in the spherical cavity immediately. Based on the gas-solid coupling relation, Assume the process is expansion in the constant pressure without heat exchange and mass lost. By the ideal gas Eq. 1

$$
\frac{P V}{T}=R n \text {. }
$$

Assume the gas is the ideal gas, that is, the process is a process with constant mass and temperature. The above equation can be simplified the following Eq. 2

$$
\begin{aligned}
& P_{1} V_{1}=P_{2} V_{2} . \\
& T \text { - gas temperature } \\
& V \text { - volume of gas } \\
& P \text { - pressure }
\end{aligned}
$$

Because the pressure of transportation pipe decreased sharply, the $\mathrm{CO}_{2}$ will expand and lead the cavity increased rapidly (see fig.8). The materials near the sphere cavity of tube wall will induce complex stress and strain field. And meanwhile, the balance equation is $\Delta V_{\text {gas }}=\Delta V_{\text {cavity }} . \Delta V_{\text {gas }}$ is expansion increment of gas volume, and $\Delta V_{\text {cavity }}$ is the expansion increment of sphere cavity. Fig.9 is the normal displacement of each point on the cavity surface.

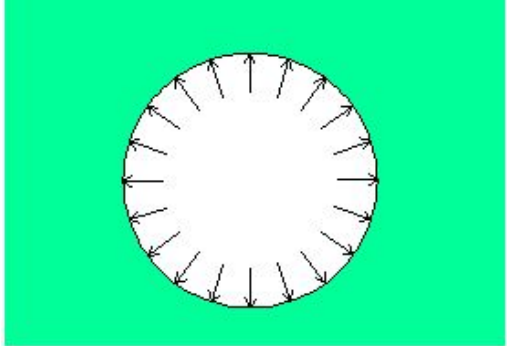

Fig.7 $\mathrm{CO}_{2}$ expansion in cavity

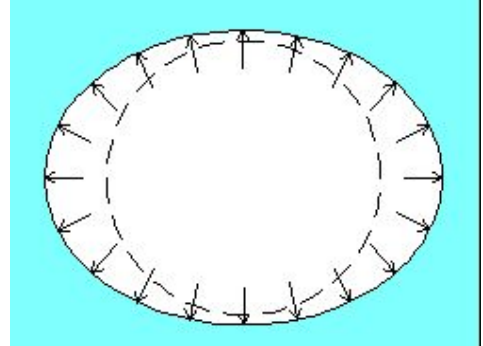

Fig. 8 Normal displacement of cavity surface

With the expansion process of the cavity surface induced by the $\mathrm{CO}_{2}$ expanding, then, the gas pressure decreased and the stress and strain of materials surrounding the cavity increased until the gas pressure of cavity $\left(p_{\text {gas }}\right)$ is equal to the reverse compressive stress $\left(p_{\text {reverse }}\right)$ induced by deformation of the surrounding materials. Fig. 10 is the balance state under the pressure of the $p_{\text {gas }}$ and $p_{\text {reverse }}$

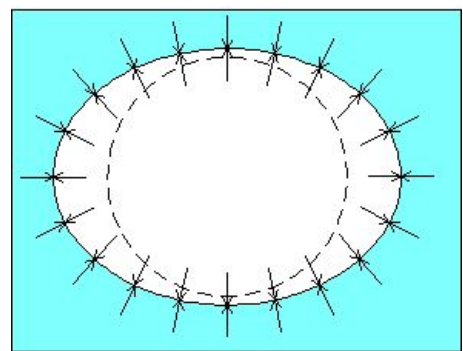

Fig.9 The balance state of $p_{\text {gas }}$ and $p_{\text {reverse }}$

When the gas expansion increment is equal to the cavity expansion, and meantime the gas pressure is equal to the normal stress induced by the deformation of exterior materials, the expansion process is at the dynamic balance state. The balance equations were described as follow Eq. 3 and Eq.4

$$
\begin{gathered}
\Delta V_{\text {gas }}=\Delta V_{\text {solid }} . \\
p_{\text {gas }}=p_{\text {reaction }} .
\end{gathered}
$$


The FEM calculation was carried based on the balance equations. Firstly, Assume $d v_{n}$ is the tiny increments of gas expansion. The gas pressure inside the cavity $\left(p_{n+1}\right)$ can be calculated by the following Eq.5 and Eq.6

$$
\begin{aligned}
& P_{n} V_{n}=P_{n+1}\left(V_{n}+d v_{n}\right) . \\
& d p_{n+1}=p_{n}-p_{n+1} .
\end{aligned}
$$

The cavity surface was applied the normal pressure increment $d_{p_{n+1}}$, and the according stress and strain field was calculated on the iterative calculations method until the pressure of gas and the cavity exterior is close to the balance. Fig. 11 and fig. 12 represent the stress field and strain field surrounding the cavity of the in-tube wall.

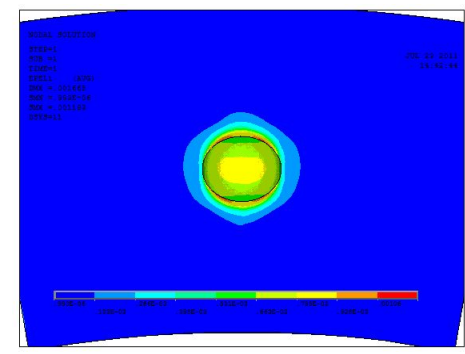

Fig.10 The stress field surrounding the cavity

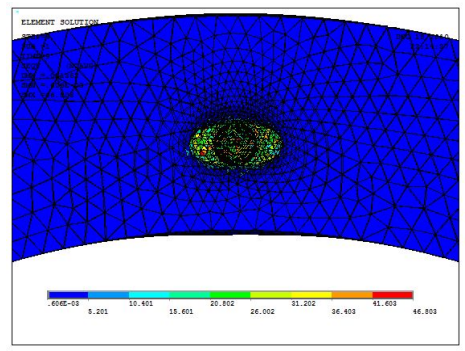

Fig.11 The strain field surrounding the cavity

The distribution laws of the stress and strain show that the micro-cavity deformation expands all around along the fiber laminates and the higher-lower expansion is lower compared to this laminates. Therefore, the cavity developed to be ellipsoid. The pipe was wound from multi-layer fiber, and therefore, the function of the top laminated materials is different with that of the bottom. The role of the bottom material of the cavity is equal to the arch, and inner layer of the tube wall is in the state of radial compressive and hoop tension. However, the top laminated material play a role of the hoop, and inner layer of the tube wall is in the state of radial and hoop compressive. Therefore, the gas expansion was restricted by the laminated materials, and the cavity evolved into ellipsoid (see fig. 13).
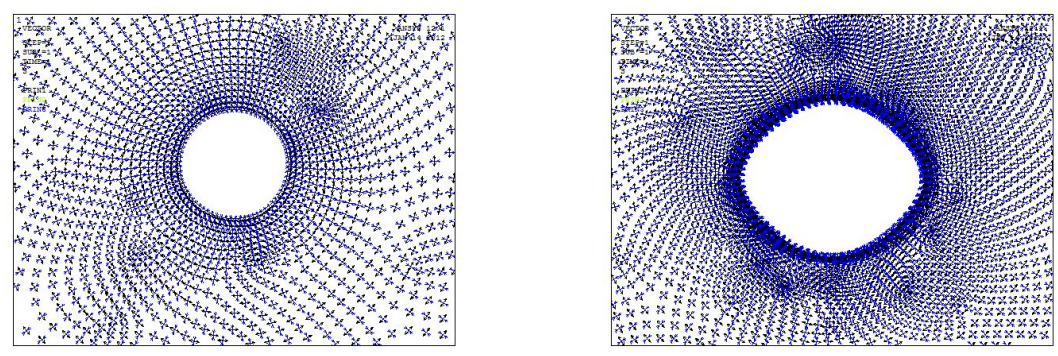

Fig.12 the vector distribution of principal stress near the cavity

Considering the cavity expansion evolved in the layer, the weakest parts were the interface of fiber and laminated resin, and especially, ends of the ellipsoid are prone to cause the stress concentration and failure of the laminated resin. And the simulated results were consistent with the experiment (shown in the fig. 2 and 3 ).

\section{Conclusion}

Using pipeline transportation, the gas pressure is very high and the transportation pressure made the tube wall in the state of radial compressive and hoop tension.

To the cavity, in-ring material at its bottom played the arch role and loaded the compressive stress and ex-ring material acting as the hoop loaded the tension stress. Loading with the gas-transport pressure, the ex-ring material was taken as a good stiffness hoop and made the cavity in stability state. 
When the operating pressure of the pipe was released sharply, the radial stress and hoop stress of all the laminates deceased or disappeared and led to the stiffness of the tube wall decreasing. Even the in-ring at the bottom of the cavity still loaded the compressive stress and the ex-ring at its top loaded with the tension stress, but the cavity will be instability, expand rapidly and then form "bomb effect" for the stiffness decreasing. The deformation of the cavity developed into ellipsoid because the weakest parts were the interface of fiber and laminated resin.

\section{Acknowledgements}

The authors are grateful to the National Natural Science Foundation of China (No. 51478144) for their financial support for this work.

\section{References}

[1] Pham DC. Shakedown Theory for Elastic Plastic Kinematic Hardening Bodies. International Journal of Plasticity, 2007,23( 7) : 1240-1259

[2] Polizzotto C. Shakedown Analysis for a Class of Strengthening Materials Within the Framework of Gradient Plasticity. International Journal of Plasticity, 2010, 26( 7) : 1050 -1069

[3] Nayebi A. Influence of Continuum Damage Mechanics on the Bree's Diagram of a Closed End Tube. Material and Design. 2010, 31: 296-305

[4] Gao BJ, Chen X, Chen G. Ratcheting and Ratcheting Boundary Study of Pressurized Straight Low Carbon Steel Pipe under Reversed bending. International Journal of Pressure Vessels and Piping, 2006,83( 2) : $96-106$

[5] Carvelli V, Cen ZZ, Liu Yet al. Shakedown Analysis of Defective Pressure Vessels by a Kinematic Approach. Archive of Applied Mechanics, 1999, 69( 9-10) : 751 -764

[6] Zhen Xiao-tao, Xuan Fu-zhen, Advances in Shakedown Design Methods for Pressure Vessels and Piping. Pressure Vessel Technology. 2010,(10): 36-45

[7] Zhu Wen-bo, Shi Jian-qiang, Lin Xing-hua. Study of Semicircle-pipe Jacket's Design by Criterions and Finite Element Analysis. Pressure Vessel Technology. 2002,19( 9):16-18 